\title{
ON SOLITON SOLUTIONS TO A CLASS OF SCHRÖDINGER-KdV SYSTEMS
}

\author{
CHUNGEN LIU AND YOUQUAN ZHENG
}

(Communicated by Yingfei Yi)

\begin{abstract}
In this paper, we consider a class of coupled nonlinear Schrödinger$\mathrm{KdV}$ systems in the whole space via the Nehari manifold method. The existence of nontrivial solutions with both of the components nonzero is obtained.
\end{abstract}

\section{INTRODUCTION}

Bound state and ground state solutions of the following coupled nonlinear Schrödinger-Korteweg-de Vries (Schrödinger-KdV for short) systems

$$
\left\{\begin{array}{l}
i f_{t}+D^{2} f=\beta f g-|f|^{2} f \\
g_{t}+D^{3} g+g D g=\frac{\beta}{2} D\left(|f|^{2}\right)
\end{array}\right.
$$

were considered by many authors, where $f=f(x, t)$ is a complex-valued function, $g=g(x, t)$ is real-valued, $(t, x) \in \mathbf{R}^{+} \times \mathbf{R}$ and $D=\frac{\partial}{\partial x}$. Systems of the form (1.1) appear as models for interactions between long and short waves in a variety of physical settings: for example, in the case of the interaction between long gravity waves and capillary waves on the surface of shallow water, in the case when the group velocity of the capillary wave coincides with the velocity of the long wave, and also for resonant ion-sound/Langmuir-wave interactions in plasmas, which reduces to (1.1) under the assumption that the ion-sound wave is unidirectional; see [9], 11, 14, 19.

Solutions for (1.1) of the form $(f(x, t), g(x, t))=\left(\exp (i \omega t) \exp (i k x) u_{1}(x-c t)\right.$, $\left.u_{2}(x-c t)\right)$ are the so-called solitary waves. Let $c=2 k$ and putting $c^{*}:=k^{2}+\omega$, one obtains the following system with cubic nonlinearity:

$$
\left\{\begin{array}{l}
-u_{1}^{\prime \prime}+c^{*} u_{1}=u_{1}^{3}-\beta u_{1} u_{2} \\
-u_{2}^{\prime \prime}+c u_{2}=\frac{1}{2} u_{2}^{2}-\frac{\beta}{2} u_{1}^{2}
\end{array}\right.
$$

Motivated by this formula, in our work we deal with the following system:

$$
\left\{\begin{array}{l}
-\Delta u+u=u^{3}-\beta u v, \quad u \in H^{1}\left(\mathbf{R}^{N}\right), \\
-\Delta v+v=\frac{1}{2}|v| v-\frac{\beta}{2} u^{2}, \quad v \in H^{1}\left(\mathbf{R}^{N}\right),
\end{array}\right.
$$

where $N=2,3$.

Systems (1.1) and (1.2) were studied in [3]. By applying the concentrationcompactness method to a constrained minimizing problem, the authors obtained

Received by the editors March 30, 2011 and, in revised form, December 11, 2011.

2010 Mathematics Subject Classification. Primary 35J10, 35J50, 35J60.

Key words and phrases. Schrödinger-KdV systems, Nehari manifold, nontrivial solution.

The first author was partially supported by NFSC (11071127, 10621101), the 973 Program of STM of China (2011CB808002) and SRFDP. 
the existence of smooth bound states for (1.2). Recently, in [4], the authors considered the Cauchy problem and the existence of bound states for coupled nonlinear Schrödinger-gKdV systems which are natural extensions of Schrödinger-KdV systems. In [2], the authors considered the following system:

$$
\left\{\begin{array}{l}
i\left(u_{t}+c_{1} u_{x}\right)+\delta_{1} u_{x x}=\alpha u v \\
v_{t}+c_{2} v_{x}+\delta_{2} v_{x x x}+\gamma\left(v^{2}\right)_{x}=\beta\left(|u|^{2}\right)_{x} .
\end{array}\right.
$$

By studying the minimizers of the energy functional subject to constraints on conserved functionals associated with symmetries of the system, they proved the existence and stability of a two-parameter family of ground states associated with a two-parameter family of symmetries. In [16], the following system was studied:

$$
\left\{\begin{array}{l}
i u_{t}+u_{x x}=\alpha u v \\
v_{t}+\gamma D v_{x}+\gamma\left(v^{2}\right)_{x}=\beta\left(|u|^{2}\right)_{x} \\
u(x, 0)=u_{0}(x), v(x, 0)=v_{0}(x)
\end{array}\right.
$$

Using the concentration compactness technique, the authors proved the existence and some property of the solitary waves for this system. In [6], the Cauchy problem for a class of Schrödinger equations, coupled with KdV equations, was considered. In [7] and [10, the authors analyzed the stability of the solitary wave for Schrödinger-KdV systems.

Let us also mention that in [1, [12] and [13, the following system was considered:

$$
\begin{cases}-\Delta u+u=u^{3}+\beta v^{2} u, & u \in H^{1}\left(\mathbf{R}^{N}\right), \\ -\Delta v+v=v^{3}+\beta u^{2} v, & v \in H^{1}\left(\mathbf{R}^{N}\right) .\end{cases}
$$

In [1, the authors dealt with this system and proved the existence of bound and ground states provided the coupling parameter is small and large, respectively. Their method is to study the functional constrained on the Nehari manifold and evaluate the Morse index of the semi-trivial solution (of the form $(0, v)$ or $(u, 0)$ with $v \neq 0$ and $u \neq 0$ ). In 12 , the authors proved the existence of a nontrivial solution for this system by minimax arguments on the Nehari manifold. In 13, the authors proved the existence of infinitely many nodal solutions for this system by the method developed in [17. Our work is based on the method used in [1]. The main result of this paper reads as:

Theorem 1.1. Assume $N=2$ or 3 and $\gamma>0$ is a constant defined in (3.7). If $|\beta|>\gamma$, problem (1.3) possesses a radial symmetric bound state with both components nonzero.

\section{Functional FRAMEWORK}

We will use variational methods to find a solution of problem (1.3). We denote the Hilbert space of all radial functions in $H^{1}\left(\mathbf{R}^{N}\right)$ by $H_{r}^{1}\left(\mathbf{R}^{N}\right)$, endowed with the norm $\|u\|^{2}=|\nabla u|_{2}^{2}+|u|_{2}^{2}$, where $|u|_{p}$ is the usual norm in the Lebesgue space $L^{p}\left(\mathbf{R}^{N}\right)$. Let $\mathcal{H}=H_{r}^{1}\left(\mathbf{R}^{N}\right) \times H_{r}^{1}\left(\mathbf{R}^{N}\right)$ and $\|(u, v)\|_{\mathcal{H}}^{2}=\|u\|^{2}+\|v\|^{2}$. Define $I: \mathcal{H} \rightarrow \mathbf{R}$ as

$$
I(u, v)=\frac{1}{2}\|(u, v)\|_{\mathcal{H}}^{2}-\frac{1}{4} \int_{\mathbf{R}^{N}} u^{4} \mathrm{~d} x-\frac{1}{6} \int_{\mathbf{R}^{N}}|v|^{3} \mathrm{~d} x+\frac{\beta}{2} \int_{\mathbf{R}^{N}} u^{2} v \mathrm{~d} x .
$$

It is easy to see that $I$ is well defined and of class $C^{2}(\mathcal{H}, \mathbf{R})$. Define the Nehari manifold as

$$
\mathcal{M}:=\{(u, v) \in \mathcal{H} \backslash(0,0):\langle\nabla I(u, v),(u, v)\rangle=0\}
$$


Lemma 2.1. For every $(u, v) \in \mathcal{H} \backslash\{(0,0)\}$, there exists a unique $\bar{t}=\bar{t}(u, v)>0$ such that $(\bar{t} u, \bar{t} v) \in \mathcal{M}$. The maximum of $g(t)=I(t u, t v)$ for $t \geq 0$ is achieved at $\bar{t}$.

Proof. Define a functional $P: \mathcal{H} \rightarrow \mathbf{R}$ as

$$
P(u, v)=\|(u, v)\|_{\mathcal{H}}^{2}-\int_{\mathbf{R}^{N}} u^{4} \mathrm{~d} x-\frac{1}{2} \int_{\mathbf{R}^{N}}|v|^{3} \mathrm{~d} x+\frac{3 \beta}{2} \int_{\mathbf{R}^{N}} u^{2} v \mathrm{~d} x .
$$

Then $(u, v) \in \mathcal{H} \backslash\{(0,0)\}$ belongs to $\mathcal{M}$ if and only if $P(u, v)=0$. Suppose that there exists $\bar{t}>0$ such that $(\bar{t} u, \bar{t} v) \in \mathcal{M}$. Then it holds that

$$
\begin{aligned}
P(\bar{t} u, \bar{t} v) & =\left\langle I^{\prime}(\bar{t} u, \bar{t} v),(\bar{t} u, \bar{t} v)\right\rangle \\
& =\bar{t}^{2}\|(u, v)\|_{\mathcal{H}}^{2}-\bar{t}^{4} \int_{\mathbf{R}^{N}} u^{4} \mathrm{~d} x-\frac{1}{2} \bar{t}^{3} \int_{\mathbf{R}^{N}}|v|^{3} \mathrm{~d} x+\frac{3 \beta}{2} \bar{t}^{3} \int_{\mathbf{R}^{N}} u^{2} v \mathrm{~d} x=0 .
\end{aligned}
$$

Hence

$$
\begin{aligned}
\varrho(\bar{t}) & :=\|(u, v)\|_{\mathcal{H}^{2}}-\bar{t}^{2} \int_{\mathbf{R}^{N}} u^{4} \mathrm{~d} x-\frac{1}{2} \bar{t} \int_{\mathbf{R}^{N}}|v|^{3} \mathrm{~d} x+\frac{3 \beta}{2} \bar{t} \int_{\mathbf{R}^{N}} u^{2} v \mathrm{~d} x \\
& =-\left(\int_{\mathbf{R}^{N}} u^{4} \mathrm{~d} x\right) \bar{t}^{2}+\left(\frac{3 \beta}{2} \int_{\mathbf{R}^{N}} u^{2} v \mathrm{~d} x-\frac{1}{2} \int_{\mathbf{R}^{N}}|v|^{3} \mathrm{~d} x\right)^{\bar{t}}+\|(u, v)\|_{\mathcal{H}^{2}}^{2}=0 .
\end{aligned}
$$

If $\int_{\mathbf{R}^{N}} u^{4} \mathrm{~d} x \neq 0$, since $\varrho(0)=\|(u, v)\|_{\mathcal{H}^{2}}^{2}>0$, then we can find a unique $\bar{t}>0$ such that (2.4) holds; that is to say, $\bar{t}(u, v) \in \mathcal{M}$. Hence, there exists a unique $\bar{t}=\bar{t}(u, v)>0$ such that $(\bar{t} u, \bar{t} v) \in \mathcal{M}$. If $\int_{\mathbf{R}^{N}} u^{4} \mathrm{~d} x=0$, then $v \neq 0$. Hence, there also exists a unique $\bar{t}=\bar{t}(u, v)>0$ such that $(\bar{t} u, \bar{t} v) \in \mathcal{M}$.

For an element $(\tilde{u}, \tilde{v}) \in \mathcal{M}$, it holds that

$$
\left\langle I^{\prime}(\tilde{u}, \tilde{v}),(\tilde{u}, \tilde{v})\right\rangle=\|(\tilde{u}, \tilde{v})\|_{\mathcal{H}}^{2}-\int_{\mathbf{R}^{N}} \tilde{u}^{4} \mathrm{~d} x-\frac{1}{2} \int_{\mathbf{R}^{N}}|\tilde{v}|^{3} \mathrm{~d} x+\frac{3 \beta}{2} \int_{\mathbf{R}^{N}} \tilde{u}^{2} \tilde{v} \mathrm{~d} x=0 .
$$

Thus we have

$$
I(\tilde{u}, \tilde{v})=\frac{1}{6}\|(\tilde{u}, \tilde{v})\|_{\mathcal{H}}^{2}+\frac{1}{12} \int_{\mathbf{R}^{N}} \tilde{u}^{4} \mathrm{~d} x
$$

and

$\|(\tilde{u}, \tilde{v})\|_{\mathcal{H}}^{2}=\int_{\mathbf{R}^{N}} \tilde{u}^{4} \mathrm{~d} x+\frac{1}{2} \int_{\mathbf{R}^{N}}|\tilde{v}|^{3} \mathrm{~d} x-\frac{3 \beta}{2} \int_{\mathbf{R}^{N}} \tilde{u}^{2} \tilde{v} \mathrm{~d} x \leq C_{1}\|(\tilde{u}, \tilde{v})\|_{\mathcal{H}}^{4}+C_{2}\|(\tilde{u}, \tilde{v})\|_{\mathcal{H}}^{3}$.

So $\|(\tilde{u}, \tilde{v})\|_{\mathcal{H}} \geq \rho$ for some $\rho>0$ and there holds $I(\tilde{u}, \tilde{v}) \geq \frac{1}{6} \rho^{2}$.

Since

$$
g(t)=\frac{t^{2}}{2}\|(u, v)\|_{\mathcal{H}}^{2}-\frac{t^{4}}{4} \int_{\mathbf{R}^{N}} u^{4} \mathrm{~d} x-\frac{t^{3}}{6} \int_{\mathbf{R}^{N}}|v|^{3} \mathrm{~d} x+\frac{\beta t^{3}}{2} \int_{\mathbf{R}^{N}} u^{2} v \mathrm{~d} x,
$$

$g(t) \rightarrow 0$ as $t \rightarrow 0, g(t) \rightarrow-\infty$ as $t \rightarrow+\infty$ and $g(\bar{t}) \geq \frac{1}{6} \rho^{2}$, so the maximum of $g(t)$ is achieved in the interior of $[0,+\infty]$. Assume that $g(\tilde{t})=\max g(t)$; then $g^{\prime}(\tilde{t})=0$. Hence $\left\langle I^{\prime}(\tilde{t} u, \tilde{t} v),(\tilde{t} u, \tilde{t} v)\right\rangle=0$, so $\tilde{t}=\bar{t}$.

Remark 2.2. Since $\left\langle P^{\prime}(u, v),(u, v)\right\rangle=-\|(u, v)\|_{\mathcal{H}}^{2}-\int_{\mathbf{R}^{N}} u^{4} \mathrm{~d} x<-\rho^{2}$ for $(u, v) \in \mathcal{M}$, $\mathcal{M}$ is a $C^{1}$ complete manifold.

Lemma 2.3. $(u, v)$ is a nontrivial critical point of $I$ if and only if $(u, v)$ is a constrained critical point of $I$ on $\mathcal{M}$.

Proof. Suppose $\left.I^{\prime}\right|_{\mathcal{M}}(u, v)=0$. By the Lagrangian multiplier method, one can see $I^{\prime}(u, v)-\omega P^{\prime}(u, v)=0$ in $\mathcal{H}$ with $(u, v) \in \mathcal{M}$. By the definition of $\mathcal{M}$, $\left\langle\left. I^{\prime}\right|_{\mathcal{M}}(u, v),(u, v)\right\rangle=-\omega\left\langle P^{\prime}(u, v),(u, v)\right\rangle=0$. From Remark 2.2. $\omega=0$. Thus $I^{\prime}(u, v)=0$ in $\mathcal{H}$. If $(u, v)$ is a non-trivial critical point of $I$, it is of course a critical point of $I$ constrained on $\mathcal{M}$. 
Lemma 2.4. I satisfies the Palais-Smale condition on $\mathcal{M}$.

Proof. Suppose that $\left\{\left(u_{n}, v_{n}\right)\right\} \subseteq \mathcal{M}$ is a Palais-Smale sequence. Then $I\left(u_{n}, v_{n}\right) \rightarrow$ $c$ and $\left.I^{\prime}\left(u_{n}, v_{n}\right)\right|_{\mathcal{M}} \rightarrow 0$ for some $c \in \mathbf{R}$. From (2.5), we can infer that $\left(u_{n}, v_{n}\right)$ is bounded in $\mathcal{H}$. So we can suppose that $\left(u_{n}, v_{n}\right) \rightarrow(u, v)$ for some $(u, v) \in \mathcal{H}$. Since $H_{r}^{1}\left(\mathbf{R}^{N}\right)$ can be compactly embedded into $L^{p}\left(\mathbf{R}^{N}\right)$ for $2<p<2^{*}$ (see [18]), we can infer that $u_{n} \rightarrow u, v_{n} \rightarrow v$ in $L^{p}\left(\mathbf{R}^{N}\right)$ for $2<p<2^{*}, u_{n} \rightarrow u, v_{n} \rightarrow v$ almost everywhere in $\mathbf{R}^{N}$. Furthermore, there hold

$$
\begin{aligned}
\int_{\mathbf{R}^{N}} u_{n}^{4} \mathrm{~d} x+\frac{1}{2} \int_{\mathbf{R}^{N}}\left|v_{n}\right|^{3} \mathrm{~d} x-\frac{3 \beta}{2} & \int_{\mathbf{R}^{N}} u_{n}^{2} v_{n} \mathrm{~d} x \\
& \rightarrow \int_{\mathbf{R}^{N}} u^{4} \mathrm{~d} x+\frac{1}{2} \int_{\mathbf{R}^{N}}|v|^{3} \mathrm{~d} x-\frac{3 \beta}{2} \int_{\mathbf{R}^{N}} u^{2} v \mathrm{~d} x
\end{aligned}
$$

and

We have

$$
\int_{\mathbf{R}^{N}} u^{4} \mathrm{~d} x+\frac{1}{2} \int_{\mathbf{R}^{N}}|v|^{3} \mathrm{~d} x-\frac{3 \beta}{2} \int_{\mathbf{R}^{N}} u^{2} v \mathrm{~d} x \geq \rho^{2} .
$$

$$
\left.I^{\prime}\left(u_{n}, v_{n}\right)\right|_{\mathcal{M}}=I^{\prime}\left(u_{n}, v_{n}\right)-\omega_{n} P^{\prime}\left(u_{n}, v_{n}\right) \rightarrow 0
$$

for a sequence of real numbers $\left\{\omega_{n}\right\}$ by the Lagrangian multiplier method. Then it holds that

$$
\left\langle\left. I^{\prime}\left(u_{n}, v_{n}\right)\right|_{\mathcal{M}},\left(u_{n}, v_{n}\right)\right\rangle=\left\langle I^{\prime}\left(u_{n}, v_{n}\right),\left(u_{n}, v_{n}\right)\right\rangle-\omega_{n}\left\langle P^{\prime}\left(u_{n}, v_{n}\right),\left(u_{n}, v_{n}\right)\right\rangle \rightarrow 0 .
$$

Since $\left\langle I^{\prime}\left(u_{n}, v_{n}\right),\left(u_{n}, v_{n}\right)\right\rangle=0$, we have $\omega_{n}\left\langle P^{\prime}\left(u_{n}, v_{n}\right),\left(u_{n}, v_{n}\right)\right\rangle \rightarrow 0$. From Remark 2.2, it implies that $\omega_{n} \rightarrow 0$. Denote $I(u, v):=\frac{1}{2}\|(u, v)\|_{\mathcal{H}}-F(u, v)-G(u, v)+$ $\beta T(u, v)$, where $F(u, v)=\frac{1}{4} \int_{\mathbf{R}^{N}} u^{4} \mathrm{~d} x, G(u, v)=\frac{1}{6} \int_{\mathbf{R}^{N}}|v|^{3} \mathrm{~d} x$ and $T(u, v)=$ $\frac{1}{2} \int_{\mathbf{R}^{N}} u^{2} v \mathrm{~d} x$. Then we have

$$
I^{\prime}\left(u_{n}, v_{n}\right)=\left(u_{n}, v_{n}\right)-F^{\prime}\left(u_{n}, v_{n}\right)-G^{\prime}\left(u_{n}, v_{n}\right)+\beta T^{\prime}\left(u_{n}, v_{n}\right)
$$

and

$$
P^{\prime}\left(u_{n}, v_{n}\right)=2\left(u_{n}, v_{n}\right)-4 F^{\prime}\left(u_{n}, v_{n}\right)-3 G^{\prime}\left(u_{n}, v_{n}\right)+3 \beta T^{\prime}\left(u_{n}, v_{n}\right) .
$$

Hence from (2.6) there holds

$$
\begin{aligned}
\left(1-2 \omega_{n}\right)\left(u_{n}, v_{n}\right)= & \left(1-4 \omega_{n}\right) F^{\prime}\left(u_{n}, v_{n}\right)+\left(1-3 \omega_{n}\right) G^{\prime}\left(u_{n}, v_{n}\right) \\
& +\left(3 \omega_{n}-1\right) \beta T^{\prime}\left(u_{n}, v_{n}\right)+o(1) .
\end{aligned}
$$

Now we prove that $F^{\prime}, G^{\prime}$ and $T^{\prime}$ are compact operators. For any $h \in H_{r}^{1}\left(\mathbf{R}^{N}\right)$, we have

$$
\begin{aligned}
\left|\left\langle T_{u}^{\prime}\left(u_{n}, v_{n}\right)-T_{u}^{\prime}(u, v), h\right\rangle\right| & =\left|\int_{\mathbf{R}^{N}}\left(u_{n} v_{n}-u v\right) h \mathrm{~d} x\right| \leq \int_{\mathbf{R}^{N}}\left|u_{n} v_{n}-u v\right||h| \mathrm{d} x \\
& \leq \int_{\mathbf{R}^{N}}\left|u_{n} v_{n}-u_{n} v\right||h| \mathrm{d} x+\int_{\mathbf{R}^{N}}\left|u_{n} v-u v\right||h| \mathrm{d} x \\
& =\int_{\mathbf{R}^{N}}\left|v_{n}-v\right|\left|u_{n}\right||h| \mathrm{d} x+\int_{\mathbf{R}^{N}}\left|u_{n}-u\right||v||h| \mathrm{d} x \\
& \leq\left|v_{n}-v\right|_{3}\left|u_{n}\right|_{3}|h|_{3}+\left|u_{n}-u\right|_{3}|v|_{3}|h|_{3} \rightarrow 0, \\
\left\langle T_{v}^{\prime}\left(u_{n}, v_{n}\right)-T_{v}^{\prime}(u, v), h\right\rangle & =\frac{1}{2} \int_{\mathbf{R}^{N}}\left(u_{n}^{2}-u^{2}\right) h \mathrm{~d} x \leq \frac{1}{2} \int_{\mathbf{R}^{N}}\left|u_{n}^{2}-u^{2}\right||h| \mathrm{d} x \\
& \leq \frac{1}{2}\left(\int_{\mathbf{R}^{N}}\left|u_{n}^{2}-u^{2}\right|^{2} \mathrm{~d} x\right)^{\frac{1}{2}}\left(\int_{\mathbf{R}^{N}}|h|^{2} \mathrm{~d} x\right)^{\frac{1}{2}} .
\end{aligned}
$$


Since $u_{n}^{4}+u^{4}-\left|u_{n}^{4}-u^{4}\right| \geq 0$, by Fatou's Lemma, we have

$$
\int_{\mathbf{R}^{N}} \liminf _{n \rightarrow+\infty}\left(u_{n}^{4}+u^{4}-\left|u_{n}^{4}-u^{4}\right|\right) \mathrm{d} x \leq \liminf _{n \rightarrow+\infty} \int_{\mathbf{R}^{N}}\left(u_{n}^{4}+u^{4}-\left|u_{n}^{4}-u^{4}\right|\right) \mathrm{d} x,
$$

and so $\lim _{n \rightarrow+\infty} \int_{\mathbf{R}^{N}}\left|u_{n}^{4}-u^{4}\right| \mathrm{d} x \rightarrow 0$. Thus $\int_{\mathbf{R}^{N}}\left|u_{n}^{2}-u^{2}\right|^{2} \mathrm{~d} x \leq \int_{\mathbf{R}^{N}}\left|u_{n}^{4}-u^{4}\right| \mathrm{d} x \rightarrow 0$ and $\left\langle T_{v}^{\prime}\left(u_{n}, v_{n}\right)-T_{v}^{\prime}(u, v), h\right\rangle \rightarrow 0$. So we have proved the compactness of $T^{\prime}$. Similarly, we have

$$
\begin{aligned}
& \left\langle F_{u}^{\prime}\left(u_{n}, v_{n}\right)-F_{u}^{\prime}(u, v), h\right\rangle=\int_{\mathbf{R}^{N}}\left(u_{n}^{3}-u^{3}\right) h \mathrm{~d} x \leq \int_{\mathbf{R}^{N}}\left|u_{n}^{3}-u^{3}\right||h| \mathrm{d} x \\
& \quad \leq\left(\int_{\mathbf{R}^{N}}\left|u_{n}^{3}-u^{3}\right|^{\frac{4}{3}} \mathrm{~d} x\right)^{\frac{3}{4}}|h|_{4} \leq\left(\int_{\mathbf{R}^{N}}\left|u_{n}^{4}-u^{4}\right| \mathrm{d} x\right)^{\frac{3}{4}}|h|_{4} \rightarrow 0, \\
& \left\langle G_{v}^{\prime}\left(u_{n}, v_{n}\right)-G_{v}^{\prime}(u, v), h\right\rangle=\frac{1}{2} \int_{\mathbf{R}^{N}}\left(\left|v_{n}\right| v_{n}-|v| v\right) h \mathrm{~d} x \\
& \quad \leq \frac{1}{2} \int_{\mathbf{R}^{N}}\left|\left(\left|v_{n}\right| v_{n}-|v| v\right)\right||h| \mathrm{d} x \\
& \quad \leq \frac{1}{2}\left(\int_{\mathbf{R}^{N}}\left(\left|v_{n}\right| v_{n}-|v| v\right)^{2} \mathrm{~d} x\right)^{\frac{1}{2}}|h|_{2} \leq\left(\int_{\mathbf{R}^{N}}\left|v_{n}^{4}-v^{4}\right| \mathrm{d} x\right)^{\frac{1}{2}}|h|_{2} \rightarrow 0 .
\end{aligned}
$$

Here, we have used the fundamental inequality $a^{\frac{q}{p}}-b^{\frac{q}{p}} \geq\left(a^{q}-b^{q}\right)^{\frac{1}{p}}$ for $a>b>0$ and $p>0, q>0$. Thus $F^{\prime}$ and $G^{\prime}$ are also compact operators.

Hence $\left(u_{n}, v_{n}\right) \rightarrow F^{\prime}(u, v)+G^{\prime}(u, v)-\beta T^{\prime}(u, v)$, so $(u, v)=F^{\prime}(u, v)+G^{\prime}(u, v)-$ $\beta T^{\prime}(u, v)$. Thus $\|(u, v)\|_{\mathcal{H}}=4 F(u, v)+3 G(u, v)-3 \beta T(u, v)$, which means $(u, v) \in$ $\mathcal{M}$.

\section{Proof of the Main theOrem}

In this section we show the existence of nontrivial solutions of (1.3) which are different from $\mathbf{u}_{0}$ and $\mathbf{u}_{1}$. Here $\mathbf{u}_{0}=(0, U), \mathbf{u}_{1}=(0,-U)$ and $U$ is the unique positive radial solution of $-\Delta u+u=\frac{1}{2} u^{2}$; see [8]. Set

$$
\gamma=\inf _{\varphi \in H \backslash\{0\}} \frac{\|\varphi\|^{2}}{\int_{\mathbf{R}^{N}} U \varphi^{2} \mathrm{~d} x} .
$$

From the Sobolev embedding theorem, $\gamma$ is a positive real number.

Theorem 3.1. $\quad$ (i) If $\beta<-\gamma$, then $\mathbf{u}_{0}$ is a saddle point of $I$ on $\mathcal{M}$. In particular, $\inf _{\mathcal{M}} I<I\left(\mathbf{u}_{0}\right)$.

(ii) If $\beta>\gamma, \mathbf{u}_{1}$ is a saddle point of $I$ on $\mathcal{M}$. In particular, $\inf _{\mathcal{M}} I<I\left(\mathbf{u}_{1}\right)$.

Let $D^{2} I_{\mathcal{M}}\left(\mathbf{u}_{0}\right)$ and $D^{2} I_{\mathcal{M}}\left(\mathbf{u}_{1}\right)$ denote the second differential of $I$ constrained on $\mathcal{M}$. Since $I^{\prime}\left(\mathbf{u}_{0}\right)=0$ and $I^{\prime}\left(\mathbf{u}_{1}\right)=0$, one has that

$$
\begin{array}{cc}
D^{2} I_{\mathcal{M}}\left(\mathbf{u}_{0}\right)[\mathbf{h}]^{2}=I^{\prime \prime}\left(\mathbf{u}_{0}\right)[\mathbf{h}]^{2}, & \forall \mathbf{h}=\left(h_{1}, h_{2}\right) \in T_{\mathbf{u}_{0}} \mathcal{M} \\
D^{2} I_{\mathcal{M}}\left(\mathbf{u}_{1}\right)[\mathbf{h}]^{2}=I^{\prime \prime}\left(\mathbf{u}_{1}\right)[\mathbf{h}]^{2} & \forall \mathbf{h}=\left(h_{1}, h_{2}\right) \in T_{\mathbf{u}_{1}} \mathcal{M} .
\end{array}
$$

Similarly, if $\mathcal{N}$ denotes the Nehari manifold relative to the functional $I_{0}: H_{r}^{1}\left(\mathbf{R}^{N}\right)$ $\rightarrow \mathbf{R}$ defined by $I_{0}(u)=\frac{1}{2}\|u\|^{2}-\frac{1}{6} \int_{\mathbf{R}^{N}}|u|^{3} \mathrm{~d} x$, i.e.,

$$
\mathcal{N}=\left\{u \in H_{r}^{1}\left(\mathbf{R}^{N}\right) \backslash\{0\}:\left\langle I_{0}^{\prime}(u), u\right\rangle=0\right\}=\left\{u \in H_{r}^{1} \backslash\{0\},\|u\|^{2}=\frac{1}{2} \int_{\mathbf{R}^{N}}|u|^{3} \mathrm{~d} x\right\},
$$


from the fact that $I_{0}^{\prime}(U)=0$ and $I_{0}^{\prime}(-U)=0$, there hold

$$
\begin{gathered}
D^{2}\left(I_{0}\right)_{\mathcal{N}}(U)[h]^{2}=I_{0}^{\prime \prime}(U)[h]^{2} \quad \forall \mathbf{h}=\left(h_{1}, h_{2}\right) \in T_{U} \mathcal{N}, \\
D^{2}\left(I_{0}\right)_{\mathcal{N}}(-U)[h]^{2}=I_{0}^{\prime \prime}(-U)[h]^{2} \quad \forall \mathbf{h}=\left(h_{1}, h_{2}\right) \in T_{-U} \mathcal{N} .
\end{gathered}
$$

Note that $\pm U$ is the only minimizer of $I_{0}$ on $\mathcal{N}$, and furthermore $I_{0}$ is nondegenerate at $U$ in the space $H_{r}^{1}\left(\mathbf{R}^{N}\right)$ since $\operatorname{Ker}_{0}^{\prime \prime}(\mathrm{U})=\operatorname{Ker}\left(-\Delta+1+\frac{1}{2} \mathrm{U}^{2}\right)=$ $\operatorname{span}\left\{\frac{\partial \mathrm{U}}{\partial \mathrm{x}_{1}}, \ldots, \frac{\partial \mathrm{U}}{\partial \mathrm{x}_{N}}\right\}$ (see [15]). Here $\frac{\partial U}{\partial x_{i}}$ is odd about the variable $x_{i}$ and every function in the space $H_{r}^{1}\left(\mathbf{R}^{N}\right)$ is even in $x_{i}$, so $\operatorname{span}\left\{\frac{\partial U}{\partial x_{1}}, \ldots, \frac{\partial U}{\partial x_{N}}\right\} \cap H_{r}^{1}\left(\mathbf{R}^{N}\right)=\{0\}$. One has that $\exists c_{0}>0$ such that

$$
\begin{gathered}
I_{0}^{\prime \prime}(U)[h]^{2}>0, \quad \forall 0 \neq h \in T_{U} \mathcal{N}, \\
I_{0}^{\prime \prime}(-U)[h]^{2}>0, \quad \forall 0 \neq h \in T_{-U} \mathcal{N} .
\end{gathered}
$$

Lemma 3.2. $\mathbf{h}=\left(h_{1}, h_{2}\right) \in T_{\mathbf{u}_{0}} \mathcal{M} \Leftrightarrow h_{2} \in T_{U} \mathcal{N}$ and $\mathbf{h}=\left(h_{1}, h_{2}\right) \in T_{\mathbf{u}_{1}} \mathcal{M} \Leftrightarrow$ $h_{2} \in T_{-U} \mathcal{N}$.

Proof. $h_{2} \in T_{U} \mathcal{N}$ if and only if $\left\langle U, h_{2}\right\rangle=\frac{3}{4} \int_{\mathbf{R}^{N}} U^{2} h_{2} \mathrm{~d} x$ and $h_{2} \in T_{-U} \mathcal{N}$ if and only if $\left\langle-U, h_{2}\right\rangle=-\frac{3}{4} \int_{\mathbf{R}^{N}} U^{2} h_{2} \mathrm{~d} x$, while $\mathbf{h} \in T_{(u, v)} \mathcal{M}$ if and only if

$\left\langle u, h_{1}\right\rangle+\left\langle v, h_{2}\right\rangle=2 \int_{\mathbf{R}^{N}} u^{3} h_{1} \mathrm{~d} x+\frac{3}{4} \int_{\mathbf{R}^{N}}|v| v h_{2} \mathrm{~d} x-\frac{3}{2} \beta \int_{\mathbf{R}^{N}} u v h_{1} \mathrm{~d} x-\frac{3}{4} \beta \int_{\mathbf{R}^{N}} u^{2} h_{2} \mathrm{~d} x$.

Hence $\mathbf{h}=\left(h_{1}, h_{2}\right) \in T_{\mathbf{u}_{0}} \mathcal{M}$ if and only if $\left\langle U, h_{2}\right\rangle=\frac{3}{4} \int_{\mathbf{R}^{N}} U^{2} h_{2} \mathrm{~d} x$ and $\mathbf{h}=$ $\left(h_{1}, h_{2}\right) \in T_{\mathbf{u}_{1}} \mathcal{M}$ if and only if $\left\langle-U, h_{2}\right\rangle=-\frac{3}{4} \int_{\mathbf{R}^{N}} U^{2} h_{2} \mathrm{~d} x$.

Proof of Theorem 3.1. If $(u, v) \in \mathcal{H}$ and $\mathbf{h}=\left(h_{1}, h_{2}\right) \in \mathcal{H}$, then one has

$$
\begin{aligned}
I^{\prime \prime}(u, v)[\mathbf{h}]^{2}= & \left\|h_{1}\right\|^{2}+\left\|h_{2}\right\|^{2}-3 \int_{\mathbf{R}^{N}} u^{2} h_{1}^{2} \mathrm{~d} x-\int_{\mathbf{R}^{N}} v h_{2}^{2} \mathrm{~d} x \\
& +\beta \int_{\mathbf{R}^{N}} v h_{1}^{2} \mathrm{~d} x+\beta \int_{\mathbf{R}^{N}} u h_{1} h_{2} \mathrm{~d} x .
\end{aligned}
$$

In particular, if $(u, v)=\mathbf{u}_{0}=(0, U)$, we get

$$
I^{\prime \prime}\left(\mathbf{u}_{0}\right)[\mathbf{h}]^{2}=\left\|h_{1}\right\|^{2}+\left\|h_{2}\right\|^{2}-\int_{\mathbf{R}^{N}} U h_{2}^{2} \mathrm{~d} x+\beta \int_{\mathbf{R}^{N}} U h_{1}^{2} \mathrm{~d} x .
$$

Take $\mathbf{h}=\left(h_{1}, 0\right) \in T_{\mathbf{u}_{0}} \mathcal{M}$; then we have

$$
I^{\prime \prime}\left(\mathbf{u}_{0}\right)\left[\left(h_{1}, 0\right)\right]^{2}=\left\|h_{1}\right\|^{2}+\beta \int_{\mathbf{R}^{N}} U h_{1}^{2} \mathrm{~d} x .
$$

By the definition of $\gamma$, we can infer that when $\beta<-\gamma$, there exists $\widetilde{h_{1}} \in H_{r}^{1}\left(\mathbf{R}^{N}\right) \backslash$ $\{0\}$ such that

So

$$
\gamma \leq \frac{\left\|\widetilde{h_{1}}\right\|^{2}}{\int_{\mathbf{R}^{N}} U{\widetilde{h_{1}}}^{2} \mathrm{~d} x}<-\beta .
$$

$$
\left.I^{\prime \prime}\left(\mathbf{u}_{0}\right)\left[\widetilde{h_{1}}, 0\right)\right]^{2}=\left\|\widetilde{h_{1}}\right\|^{2}+\beta \int_{\mathbf{R}^{N}} U{\widetilde{h_{1}}}^{2} \mathrm{~d} x<0 .
$$

From (3.8) and (3.11), we can infer that $\mathbf{u}_{0}$ is a saddle point.

If $(u, v)=\mathbf{u}_{1}=(0,-U)$, we get

$$
I^{\prime \prime}\left(\mathbf{u}_{1}\right)[\mathbf{h}]^{2}=\left\|h_{1}\right\|^{2}+\left\|h_{2}\right\|^{2}+\int_{\mathbf{R}^{N}} U h_{2}^{2} \mathrm{~d} x-\beta \int_{\mathbf{R}^{N}} U h_{1}^{2} \mathrm{~d} x .
$$


Take $\mathbf{h}=\left(h_{1}, 0\right) \in T_{\mathbf{u}_{1}} \mathcal{M}$. We have

$$
I^{\prime \prime}\left(\mathbf{u}_{1}\right)\left[\left(h_{1}, 0\right)\right]^{2}=\left\|h_{1}\right\|^{2}-\beta \int_{\mathbf{R}^{N}} U h_{1}^{2} \mathrm{~d} x .
$$

By the definition of $\gamma$, we can infer that when $\beta>\gamma$, there exists $\widetilde{h_{2}} \in H_{r}^{1}\left(\mathbf{R}^{N}\right) \backslash\{0\}$ such that

$$
\gamma \leq \frac{\left\|\widetilde{h_{2}}\right\|^{2}}{\int_{\mathbf{R}^{N}} U{\widetilde{h_{2}}}^{2} \mathrm{~d} x}<\beta .
$$

So

$$
\left.I^{\prime \prime}\left(\mathbf{u}_{1}\right)\left[\widetilde{\left(h_{2}\right.}, 0\right)\right]^{2}=\left\|\widetilde{h_{2}}\right\|^{2}-\beta \int_{\mathbf{R}^{N}} U{\widetilde{h_{2}}}^{2} \mathrm{~d} x<0 .
$$

From (3.9) and (3.12), $\mathbf{u}_{1}$ is a saddle point.

The following lemma is a direct consequence of Theorem 3.1

Lemma 3.3. (i) If $\beta>\gamma$, then I has a global minimum $(\tilde{u}, \tilde{v})$ on $\mathcal{M}$ and $I(\tilde{u}, \tilde{v})<I\left(\mathbf{u}_{1}\right)=\inf _{\mathcal{N}} I_{0}$.

(ii) If $\beta<-\gamma$, then I has a global minimum $(\tilde{u}, \tilde{v})$ on $\mathcal{M}$ and $I(\tilde{u}, \tilde{v})<I\left(\mathbf{u}_{0}\right)=$ $\inf _{\mathcal{N}} I_{0}$.

Proof. By the Ekeland variational principle (see [5]) and Lemma 2.4, we can infer that $\inf I$ is achieved at some $(\tilde{u}, \tilde{v}) \neq 0$. Moreover, if $\beta<-\gamma$, Theorem [3.1(i) implies that $I(\tilde{u}, \tilde{v})<I\left(\mathbf{u}_{0}\right)$. If $\beta>\gamma$, Theorem 3.1(ii) implies that $I(\tilde{u}, \tilde{v})<$ $I\left(\mathbf{u}_{1}\right)$. From the fact that $I\left(\mathbf{u}_{0}\right)=I_{0}(U)=\inf _{\mathcal{N}} I_{0}=I_{0}(-U)=I\left(\mathbf{u}_{1}\right)$, the proof is completed.

Proof of Theorem 1.1. By Lemma 3.3. we can get a minimizer $(\tilde{u}, \tilde{v})$ of the problem $\inf _{\mathcal{M}} I$ which is a solution of (1.3). We need to prove $\tilde{u} \neq 0$ and $\tilde{v} \neq 0$. Otherwise, if $\tilde{\tilde{v}}=0 \in H_{r}^{1}\left(\mathbf{R}^{n}\right)$, from equation (1.3), we see also that $\tilde{u}=0$. This is impossible since $(0,0) \notin \mathcal{M}$. If $\tilde{u}=0, \tilde{v} \in \mathcal{N}$, then $I_{0}(\tilde{v})=I(\tilde{u}, \tilde{v}) \leq I(0, u)=I_{0}(u)$ for any $u \in \mathcal{N}$, so $\tilde{v}$ achieves the minimum of $I_{0}$ on $\mathcal{N}$. Thus we have

$$
I(\tilde{u}, \tilde{v})=I_{0}(\tilde{v})=\inf _{\mathcal{N}} I_{0}
$$

This contradicts Lemma 3.3

\section{REFERENCES}

[1] A. Ambrosetti and E. Colorado, Standing waves of some coupled nonlinear Schrödinger equations, J. London Math. Soc., 75(2)(2007), 67-82. MR 2302730(2008f:35369)

[2] J. Albert and J. Angulo Pava, Existence and stability of ground-state solutions of a Schrödinger-KdV system, Proceedings of the Royal Society of Edinburgh, 133A(2003), 9871029. MR2018323 (2005f:35269)

[3] J. Dias, M. Figueira and F. Oliveira, Existence of bound states for the coupled Schrödinger$K d V$ system with cubic nonlinearity, Comptes Rendus Mathematique, 348(2010), 19-20, 1079-1082. MR2735011 (2011g:35348)

[4] J. Dias, M. Figueira and F. Oliveira, Well-posedness and existence of bound states for a coupled Schrödinger-gKdV system, Nonlinear Analysis, 73 (2010), 2686-2698. MR2674102 (2011g:35371)

[5] I. Ekeland, On the variational principle, Journal of Mathematical Analysis and Applications, 47(1974), 324-353. MR0346619 (49:11344) 
[6] B. Guo, Existence and uniqueness of the global solution of the Cauchy problem and the periodic initial value problem for a class of coupled systems of KdV-nonlinear Schrödinger equations. Acta Math. Sinica, 26(1983), 513-532 (Chinese). MR747175 (86e:35132)

[7] B. Guo and Y. Wu, Orbital stability of solitary waves for the nonlinear derivative Schrödinger equation, J. Diff. Eqns., 123(1995), 35-55. MR.1359911 (96k:35166)

[8] M. K. Kwong, Uniqueness of positive solutions of $\Delta u-u+u^{p}=0$ in $\mathbf{R}^{n}$, Arch. Rat. Mech. Anal., 105(1989), 243-266. MR969899 (90d:35015)

[9] T. Kawahara, N. Sugimoto and T. Kakutani, Nonlinear interaction between short and long capillary-gravity waves, J. Phys. Soc. Jpn., 39(1975), 1379-1386.

[10] L. Chen, Orbital stability of solitary waves of the nonlinear Schrödinger-KdV equation, Partial Diff. Eqns., 12(1999), 11-25. MR.1681850 (2000d:35212)

[11] V. Makhankov, On stationary solutions of the Schrödinger equation with a self-consistent potential satisfying Boussinesq's equation, Phys. Lett. A, 50(1974), 42-44.

[12] L. A. Maia, E. Montefusco and B. Pellacci, Positive solutions of a weakly coupled nonlinear Schrödinger system, J. Diff. Equations, 229(2006), 743-767. MR2263573 (2007h:35070)

[13] L. A. Maia, E. Montefusco and B. Pellacci, Infinitely many nodal solutions for a weakly coupled nonlinear Schrödinger system, Communications in Contemporary Mathematics, 10(5)(2008), 651-669. MR2446894(2009m:35108)

[14] K. Nishikawa, H. Hojo, K. Mima and H. Ikezi, Coupled nonlinear electron-plasma and ionacoustic waves, Phys. Rev. Lett., 33(1974), 148-151.

[15] W. Ni and I. Takagi, Locating the peaks of least energy solutions to a semilinear Neumann problem, Duke Math. J., 70(1993), 247-281. MR1219814(94h:35072)

[16] J. Angulo and J. F. Montenegro, Existence and evenness of solitary-wave solutions for an equation of short and long dispersive waves, Nonlinearity, 13(2000), 1595-1611. MR 1781810 (2001e:35142)

[17] S. Terracini and G. Verzini, Solutions of prescribed number of zeros to a class of superlinear ODE's systems, Nonlinear Differential Equations Appl., 8(2001), 323-341. MR 1841262 (2002f:34038)

[18] M. Willem, Minimax Theorems, Birkhäuser, Boston, 1996. MR1400007 (97h:58037)

[19] N. Yajima and J. Satsuma, Soliton solutions in a diatomic lattice system, Prog. Theor. Phys., $62(1979), 370-378$

School of Mathematical Sciences and LPMC, Nankai University, Tianjin 300071 , People's Republic of China

E-mail address: liucg@nankai.edu.cn

School of Mathematical Sciences, Nankai University, Tianjin 300071, People's Republic of ChinA

E-mail address: zhengyq@mail.nankai.edu.cn 